\section{Limplieation des patients et usagers dans les évaluations condutes par la Haute Autortié de Santé}

Chantal Bélorgey

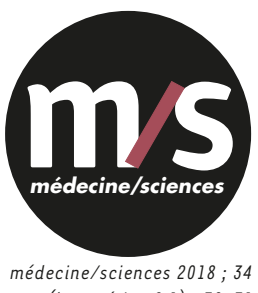

(hors série $n^{\circ} 1$ ) : $30-31$
> La HAS a pour mission de donner un avis sur les produits de santé médicaments, dispositifs médicaux - et actes professionnels en vue de leur remboursement et de la négociation des prix.

La parole des patients est donc fondamentale. Elle a plusieurs finalités. La première est démocratique, car les patients sont les premiers concernés. Ils participent également, comme tous les citoyens, au financement des produits de santé. La deuxième finalité est scientifique. On peut parler d' «évidence patient » au même titre que d'évidence clinique ou économique, car le patient est expert de sa propre maladie. Il peut exprimer la réalité de la maladie, sa vie usuelle et ses préférences. Il peut rappeler le parcours de soins aux membres des commissions de la HAS et aux experts scientifiques.

La HAS a mis des dispositifs en place dès sa précédente incarnation, I'ANAES. Elle fait intervenir les patients et usagers à différents niveaux dans les évaluations qu'elle mène sur les produits de santé : au sein de la Commission de la transparence, la Commission Nationale d'Évaluation des Dispositifs Médicaux et des technologies de Santé (CNEDIMTS), qui évalue les dispositifs médicaux, et la CE\&SP qui mène notamment les évaluations médico-économiques. Ils sont dorénavant systématiquement des membres experts dans nos groupes de travail. Ils peuvent être entendus par les commissions. Troisièmement, toute association de patients peut contribuer à l'évaluation d'un produit de santé. Cette contribution se fait sur la base d'un questionnaire établi au niveau international.

Je considère cette expérimentation comme un succès par la quantité, mais surtout la qualité des contributions. Les associations ont recouru à des questionnaires envoyés à leurs adhérents, ont effectué du data mining sur des forums, ont demandé à quelques patients d'intervenir eux-mêmes dans certaines maladies extrêmement rares. Ainsi, les méthodes utilisées sont extrêmement fouillées, bien qu'elles ne soient pas écrites ni théorisées, et que leur prise en compte ne soit pas aujourd'hui organisée comme celle d'un essai clinique.

Les associations de patients peuvent également intervenir sur le programme de travail de la HAS en proposant des sujets. Elles disposent également depuis la loi de modernisation de notre système de santé, d'un droit d'alerte : elles peuvent saisir la HAS sur tout fait ayant des incidences importantes sur la santé et relevant des compétences de la HAS. Nous avons reçu cinq droits d'alerte depuis la mise en place de ce droit en 2016. La majorité concerne des produits de santé. Dans ce cas, nous travaillons en lien avec l'ANSM, s'il s'agit d'alertes de sécurité et d'information des patients.

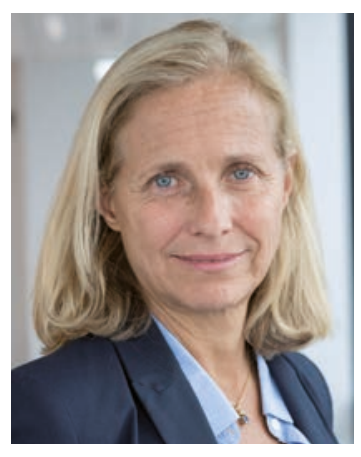

Directrice de l'évaluation médicale, économique et de santé publique, Haute Autorité de Santé, 5 , avenue du Stade de France, 93250 Saint-Denis, France. c.belorgey@has-sante.fr

En outre, nous pouvons faire intervenir les patients en amont des demandes de remboursement des produits, notamment dans les avis scientifiques menés au niveau européen avec EUnetHTA. Enfin, le collège de la HAS comprend un représentant d'associations de patients, et l'un des axes du projet stratégique que la HAS développe vise la participation des associations de patients dans nos travaux.

L'implication des patients dans les travaux de la HAS pourrait être encore plus systématique car ancrée dans la culture des chefs de projet de la HAS, au point que la question de cette participation ne se pose même plus. En amont, nous devons désormais exiger, en collaboration avec I'ANSM et l'EMA, des données de qualité de vie et de préférence patient dans les dossiers déposés par les industriels. En aval, les associations de patients ont toute leur place dans les évaluations et les études que nous menons sur les données en vie réelle.

La contribution des associations de patients est désormais un élément à part entière de l'évaluation des dossiers. En revanche, elle peut être complexe pour les associations elles-mêmes, notamment parce que les délais sont contraints. II faut former les associations à nos méthodes et procédures. De notre côté, il importe de préciser la manière dont nous valorisons et prenons en considération cette contribution, en nous inspirant des méthodes SHS (Sciences de l'Homme et de la Société). Enfin, nous devons accroître la transparence. Les contributions de patients seront publiées, notamment parce qu'elles peuvent être utiles, par leur qualité, aux associations qui n'osent pas encore répondre. II en va également de la transparence sur les liens d'intérêts. Enfin, certaines associations de patients revendiquent d'avoir 
accès aux dossiers déposés par les laboratoires. Certaines agences le font; nous devons y réfléchir. Une dernière remarque : aujourd'hui, nous devons demander l'accord des laboratoires pour mettre des informations sur le site Internet et permettre aux associations de contribuer à nos travaux. Ce sujet mérite d'être discuté avec les industriels. $\diamond$
Place of the expert patient in the assessments performed by the Haute Autorité de Santé

\section{LIENS D’INTÉRÊT}

L'auteur déclare n'avoir aucun lien d'intérêt concernant les données publiées dans cet article.

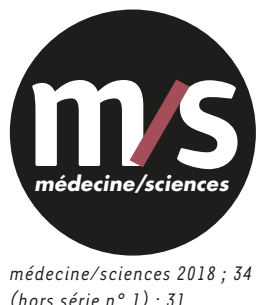

(hors série $n^{\circ} 1$ ) : 31

\section{L'implieation du malade expert dans les évaluations : le point de vue des industriels}

Catherine Raynaud
> Nous soutenons l'implication des patients dans toutes les étapes de l'évaluation des produits de santé, car nous sommes convaincus que seuls les représentants des associations permettent d'avoir une représentation sociale de la maladie. Disposer du vécu de ces malades permet de mettre l'accent sur la qualité de vie. La définition même des termes «bénéfice », «valeur » ou « risque » commence à s'élargir pour prendre en compte l'avis des malades. L'importance de la galénique a également été mise en avant : elle a un impact sur la qualité de vie et l'observance et donc sur l'efficacité.

Nous discutions, il y a dix ans, avec des professionnels de santé pour avoir l'expérience des patients. Aujourd'hui, pour mettre en place un essai clinique, l'équipe à l'origine du protocole doit le présenter à un comité de validation en interne. Ce dernier lui demande notamment si elle a travaillé avec une association de patients. Tous s'accordent à dire que cette procédure a largement amélioré l'efficacité et la qualité de la recherche.

Les industriels ont peu de visibilité sur les contributions dans le cadre de l'évaluation de la Commission de la transparence : nous devons lire les comptes rendus pour savoir s'il en existe. Dans un cas, nous avons précisé que le produit avait eu un impact fort sur la qualité de vie et l'organisation de la prise en charge. Trois autres produits ne mentionnaient rien de tel. La question était alors de savoir si la contribution ne ressortait pas au compte rendu ou si aucune association de patient n'avait pu être impliquée. La question est alors de savoir que faire dans le cas où il n'existe pas d'associations sur une pathologie donnée. Nous nous accordons tous sur la nécessité de prendre en compte les avis, de prévoir une procédure standardisée et une voie de recours. L'industriel doit effectivement donner son accord à la publication des données, mais cette autorisation est octroyée dans plus de $90 \%$ des cas pour les médicaments. Le Leem travaille également en ce sens. Un paragraphe dédié nous paraît un minimum, complété si possible

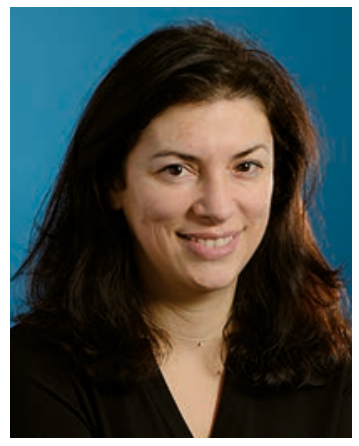

Directeur des Affaires Publiques, Pfizer, 23-25, avenue du Dr Lannelongue, 75014 Paris, France. catherine.raynaud@pfizer.com

par une audition, pour mettre l'accent sur la parole du patient et caractériser le bénéfice.

II faut également des critères d'évaluation adaptés quand nous montrons qu'un traitement a un impact positif sur la qualité de vie, l'observance, l'organisation des soins ou une réduction du diagnostic dans le temps.

Enfin, nous devons nous assurer du bon usage du produit quand il est commercialisé. Nous sommes convaincus de la nécessité d'une concertation avec les associations de patients. II faut développer une approche d'ensemble pour déterminer la bonne formation et la bonne information, apporter des outils et des solutions adaptés à l'accompagnement.

La place réservée au patient évolue et se renforce, mais elle exige encore un cadre collaboratif fort. $\diamond$

Place of the expert patient when assessing medicinal products: the industry perspective

\section{LIENS D'INTÉRÊT}

L'auteur déclare n'avoir aucun lien d'intérêt concernant les données publiées dans cet article. 